\title{
Uma análise sobre a disposição dos serviços de cuidados paliativos no Brasil
}

\author{
An analysis on the disposal of palliative care services in Brazil
}

Poliana YabuUti ${ }^{1}$

Gustavo Monteiro de Jesus ${ }^{1}$

Maria Alice Santos Rocha ${ }^{\mathrm{I}}$

KAREN EgêA BACO ${ }^{\mathrm{I}}$

Maria Olivia Costa Parra

REBoLO

${ }^{\mathrm{I}}$ Universidade Mogi das Cruzes

(UMC), Mogi das Cruzes/SP - Brasil
Resumo - Introdução: Cuidados Paliativos (CP) são um conjunto de práticas que visam a prevenção e o alívio do sofrimento de indivíduos (e de seus familiares) que enfrentam doenças ou condições que ameacem a vida. Inicialmente utilizados em pacientes em estado terminal, hoje os CP buscam intervir do diagnóstico ao luto. Contudo, mesmo avanços sendo feitos nessa área, o cenário ainda é longe do ideal. Objetivo: Realizar um levantamento sobre os serviços de saúde especializados em CP no Brasil, com atenção na assistência domiciliar. Métodos: Estudo descritivo, exploratório, de abordagem quantitativa, utilizando-se de dados disponíveis pela Academia Nacional de Cuidados Paliativos e pelo Cadastro Nacional dos Estabelecimentos de Saúde, os quais foram organizados, analisados e discutidos com publicações científicas sobre a temática. Resultados: Foram identificadas 164 instituições especializados em CP, com maior concentração na região Sudeste e menor no Norte, sendo $20 \%$ do total com atenção domiciliar. Sobre essa última, houve crescimento expressivo nos últimos cinco anos, passando de 3.925 para 6.139 estabelecimentos, com destaque para as Regiões Sudeste e Nordeste. Conclusão: Os CP são vistos como recursos inovadores e promissores acerca dos seus benefícios. No entanto, sua prática ainda é restrita a poucas realidades no Brasil e, clama pela necessidade de investimentos, ampliação e diversificação.

Palavras-chave: Cuidado paliativo; Assistência domiciliar; Home Care; SERviços DE SAÚde.

ABstraCt - Introduction: Palliative Care (PC) is a set of practices aimed at preventing and alleviating the suffering of individuals (and their families) who face life-threatening illnesses or conditions. Initially used in terminally ill patients, today PC seek to intervene from diagnosis to grief. However, even though advances are being made in this area, the scenario is still far from ideal. Objective: To carry out a survey on health services specialized in PC in Brazil, with attention to home care. METHODS: a descriptive, exploratory study with a quantitative approach, using data available from the Academia Nacional de Cuidados Paliativos and the Cadastro Nacional dos Estabelecimentos de Saúde, which were organized, analyzed and discussed with scientific publications on the subject. Results: 164 institutions specialized in PC were identified, with a greater concentration in the Southeast region and lower in the North, with $20 \%$ of the total with home care. Regarding the latter, there has been significant growth in the last five years, from 3,925 to 6,139 establishments, with emphasis on the Southeast and Northeast regions. CONCLUSION: PCs are seen as innovative and promising resources about their benefits. However, its practice is still restricted to few realities in Brazil and, calls for the need for investments, expansion and diversification.

Keywords: Palliative Care; Health Service, Home Care. 


\section{INTRODUÇÃO}

De acordo com a definição da Organização Mundial da Saúde (OMS) ${ }^{1}$ de 2002, "Cuidado Paliativo (CP) é uma abordagem que promove a qualidade de vida de pacientes e seus familiares, que enfrentam doenças que ameacem a continuidade da vida, através da prevenção e alívio do sofrimento. Requer a identificação precoce, avaliação e tratamento da dor e outros problemas de natureza física, psicossocial e espiritual". Segundo González², o termo cuidado (do latim cogitare) indica justamente a reflexão sobre ideias, sentimentos, fatos e circunstâncias sobre o presente, passado e futuro. Já o termo paliativo (do latim pallium, manto) significa ação de cobrir, proteger. Por conseguinte, quando as ações de apoio já não podem mais alterar o curso da doença, fazem florescer essa nova concepção de atendimento e prestação de serviço, cujo principal objetivo é aliviar o sofrimento e melhorar a qualidade de vida dos pacientes. ${ }^{3}$

Originalmente, o CP centrava-se na fase de avanço final das doenças, mas hoje se extendeu do diagnóstico até o luto, podendo ser indicado em qualquer fase de evolução da doença/condição (pacientes oncológicos, portadores de doenças crônico-degenerativas, síndromes demenciais, falências funcionais, vítimas de acidentes, entre outros), durando meses ou até anos. ${ }^{4}$ Desse modo, no momento que a paliação se torna imprescindível para que o paciente receba os cuidados essenciais e suporte de vida, o cuidador deve desenvolver a capacidade de observação, e compreensão das necessidades do enfermo. ${ }^{5}$ Aliado a isto, todos os envolvidos no processo deverão ser acolhidos e contemplados por uma equipe multidisciplinar (médico, enfermeiro, assistente social, farmacêutico, fisioterapeuta, fonoaudiólogo, nutricionista, psicólogo, terapeuta ocupacional, voluntários e religiosos). ${ }^{6}$

Porém mesmo o $\mathrm{CP}$ sendo algo tão benéfico para a população, estamos longe do ideal. Segundo a $\mathrm{OMS}^{3}$, dos 58 milhões de mortes por ano no mundo, 34 milhões são por doenças crônico-degenerativas, e destes, cerca de $70 \%$ ocorrem em ambiente hospitalar, e em sua maioria, em Unidades de Terapia Intensiva (UTI). O despreparo dos serviços para o atendimento em casos de doenças incuráveis e que levam à incapacidade e alta dependência é a realidade de muitos países como o Brasil, onde infelizmente a maioria dos hospitais não possui uma diretriz sobre como cuidar de pacientes que estejam com um quadro terminal, tampouco estão preparados para apoiar seus familiares. ${ }^{3,7}$

Por conseguinte, a impossibilidade de cura não significa que não haja mais o que ser feito ou desenvolvido, mas o que muda é o enfoque da assistência que agora se volta aos cuidados das necessidades do enfermo e de sua família. O presente estudo propôs uma reflexão acerca dos serviços de assistência paliativa no Brasil e sua abordagem frente à tradicional medicina em vigência uma vez que a terapêutica renovada busca trabalhar cada etapa com dignidade, autonomia e menor sofrimento possível, facilitando assim o enfrentamento e aceitação do processo degenerativo da doença e de sua terminalidade para o paciente, família e profissionais envolvidos. Desse modo, o 
objetivo do estudo foi realizar um levantamento sobre a evolução e disposição dos serviços de saúde especializados em cuidados paliativos no Brasil, com atenção na assistência domiciliar.

\section{Métodos}

Trata-se de um estudo descritivo, exploratório, de abordagem quantitativa, cujos dados secundários foram coletados nos meses de maio e junho de 2018. Os mesmos foram extraídos dos websites da Academia Nacional de Cuidados Paliativos (ACNP) e do Cadastro Nacional dos Estabelecimentos de Saúde (CNES). ${ }^{1,8}$

Sobre as informações obtidas pela ANCP, foram considerados os serviços que contemplavam três grupos: a) a assistência hospitalar; b) ambulatório; c) assistência domiciliar (AD). Foram categorizados como público ou privado. Para esse último, foram inclusas as variáveis "privado", "particular", "público e privado", "com SUS", "filantrópico", "cooperativa" e "convênio/operadora de saúde", ao qual não foram destrinchadas pela fonte explorada.

Em seguida, foi realizada uma busca na internet dos sites dos estabelecimentos identificados na página da ANCP a fim de encontrar publicações referentes à prática de atenção em domicílio.

Quanto à análise da $\mathrm{AD}$, foram utilizados dados disponíveis pelo CNES, pelo qual buscou-se a classificação "Serviços de atenção domiciliar" e, a partir disso, foram inclusas as categorias "Internação domiciliar", "Assistência domiciliar", "Equipe Multiprofissional de Atenção Domiciliária" (EMAD), "Equipe Multiprofissional de Apoio" (EMAP). E então para identificar os prestadores do Programa Melhor em Casa (PMC), selecionou-se os estabelecimentos dispondo de "Equipe EMAD" pelo menos.

Após essa etapa, os dados foram organizados em uma planilha, da qual originou-se a elaboração de gráficos e procedeu-se a análise e comparação das informações obtidas com as publicações científicas sobre a temática.

Considerando que se trata de pesquisa em fonte de dados secundários e de domínio público, não houve necessidade de submeter o projeto à avaliação de comitê de éti$\mathrm{ca}$ em pesquisa com seres humanos.

\section{RESUltados E Discussão}

Com relação à prática paliativa, fez-se um levantamento sobre os estabelecimentos de saúde especializados em CP reconhecidos pela ANCP, do qual foram identificadas 164 unidades, sendo 94 de caráter exclusivo público e 70 privados e com maior concentração na região Sudeste e menor na região Norte (Gráfico 1). 


\section{Gráfico 1 - Distribuição de serviços especializados em CP, segundo ANCP - 2018}

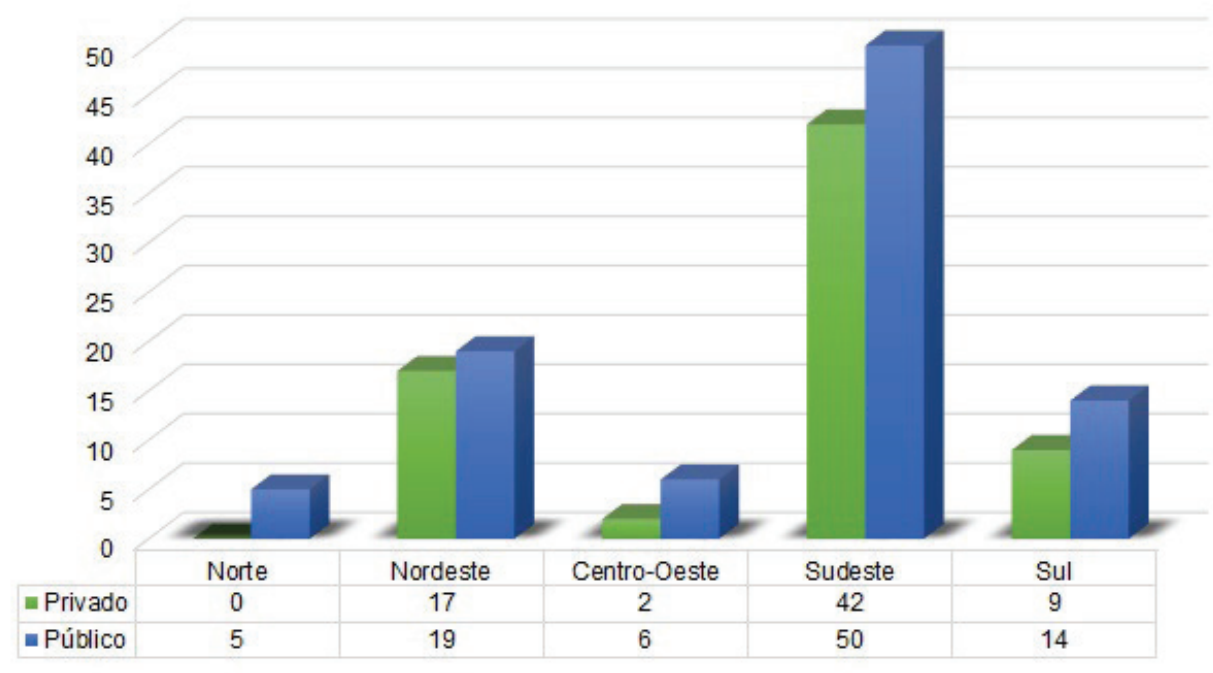

Autoria própria

Dentre os que disponibilizavam de assistência domiciliar foram encontradas 32 instituições, instaladas principalmente nas regiões Sudeste e Sul (Gráfico 2), e majoritariamente de caráter privado.

\section{Gráfico 2 - Distribuição serviços de saúde especializados em $\mathrm{CP}$ em domicilio por divisão regional no Brasil, sendo ANCP - 2018}

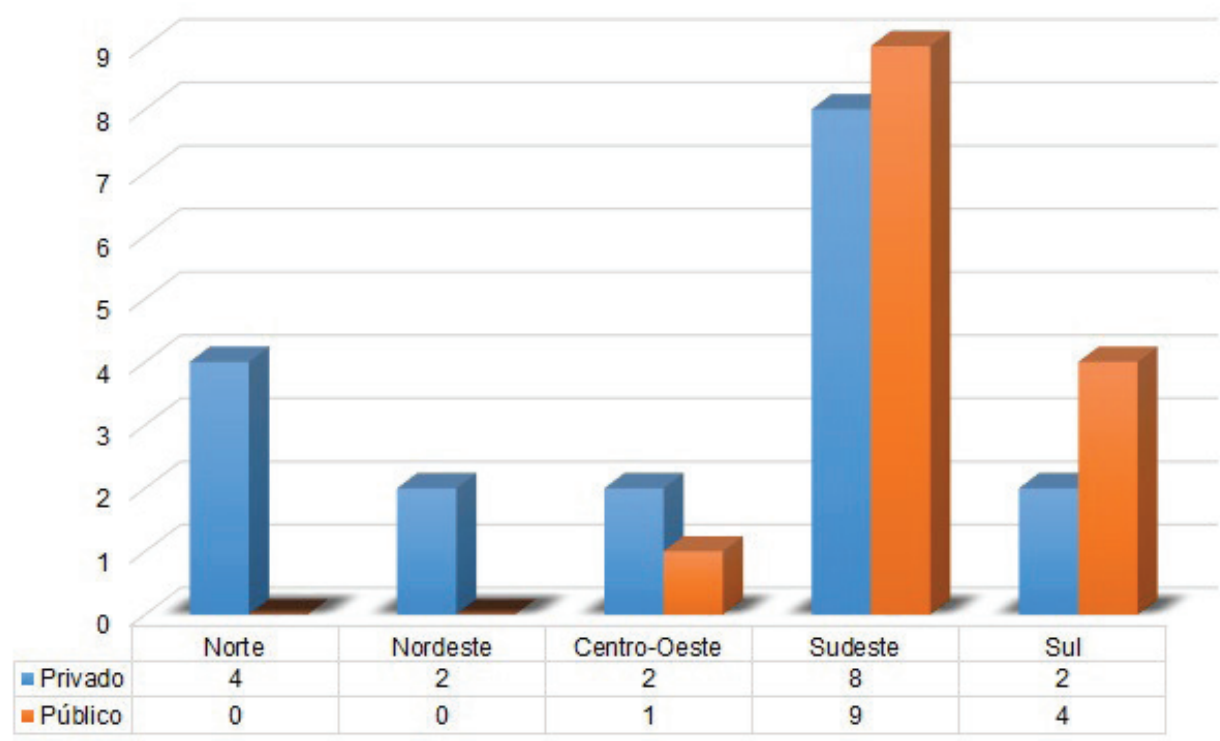

Autoria própria 
A fim de avaliar a disposição da assistência em AD no Brasil, com base no CNES observou-se um avanço expressivo nos últimos anos, passando de 3.925 para 6.139 estabelecimentos (Gráfico 3), cuja distribuição territorial é representada no Gráfico 4.

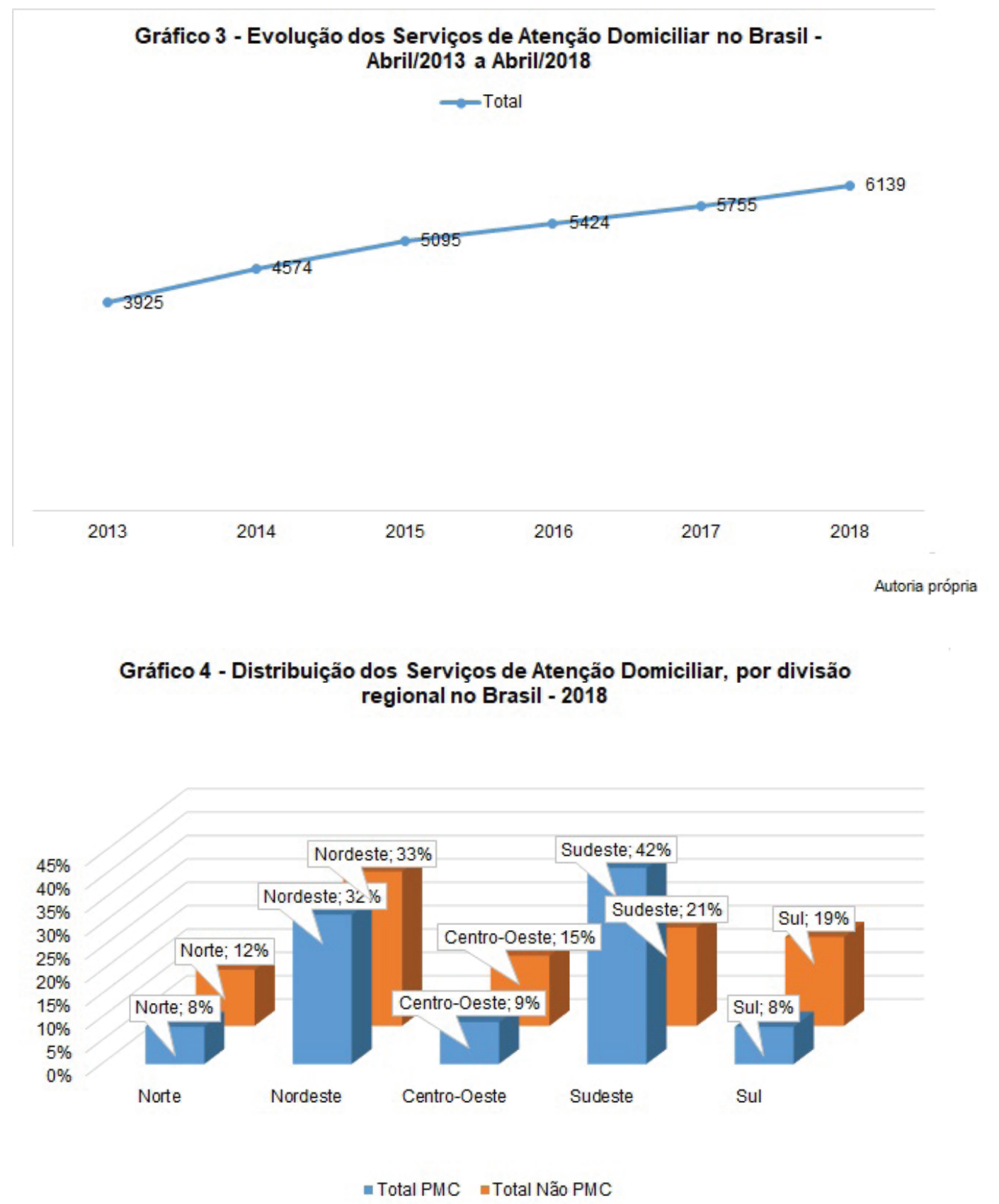

Autoria própria 
O fato dos serviços de CP concentrarem-se na região Sudeste pode estar relacionado aos aspectos socioeconômicos, nos quais o Produto Interno Bruto desta região é o mais expressivo do país e, portanto, disponibiliza os melhores recursos humanos e de financiamentos. Já o Nordeste vem apresentando expressiva participação econômica no país, tornando-se alvo de investimentos por parte do governo, enquanto o Norte é visto como uma região com dificuldades de acesso e alcance de recursos para investimento. ${ }^{9}$

Temel e colaboradores ${ }^{10}$ compararam a assistência provida no modelo tradicional e no modelo paliativo e mostraram que o grupo que recebeu cuidados paliativos precoces apresentou melhor autoavaliação de qualidade de vida. Além desse benefício, os pacientes viveram, em média, três meses mais que o outro grupo, comprovando então a eficácia dessa terapêutica. Pois, de fato, a paliação é encarada como mecanismo terapêutico para mitigar os problemas decorrentes da enfermidade, e como qualquer outro tipo de assistência, pode ser aplicada em nível hospitalar (pronto atendimento, unidade de internação, UTI), hospital-dia, hospice, ambulatorialmente e, quando possível, a assistência domiciliar (AD) ou home care. Nesse último, a proposta visa a redução no tempo de permanência do paciente internado e retorno ao seu ambiente habitual e familiar, mas sem perder a qualidade do atendimento, ao promover a sua integração, o autocuidado e a reabilitação, melhorando assim as condições psicológicas do paciente, além de diminuir os riscos de infecções hospitalares e possíveis complicações resultantes das internações prolongadas. ${ }^{10}$

No Brasil, a abordagem domiciliar se iniciou em 1963, com o Serviço de Assis- tência Domiciliar do Hospital de Servidores Públicos do Estado de São Paulo (HSPE) que funciona até hoje, e a partir daí vieram o Programa de Agentes Comunitários (PACS) em 1991, o Programa de Saúde da Família (PSF) em 1994, além de iniciativas de Prefeituras Municipais e Hospitais Universitários e, sobretudo, as empresas privadas que impulsionaram no seguimento. ${ }^{7}$ Então, em 2011 foi instituída a Política Nacional de Atenção Domiciliar no Serviços Único de Saúde (SUS), contemplando em uma de suas modalidades o Programa Melhor em Casa (PCM), aplicado em nível secundário por Equipes Multiprofissionais de Atenção Domiciliária (EMAD), incluindo médico, enfermeiro e fisioterapeuta para pacientes em condições agudas, assim como atendendo a demanda de pacientes que necessitam de uso contínuo de oxigenioterapia, suporte ventilatório não invasivo, paracentese ou diálise peritoneal junto à Equipe Multiprofissional de Apoio (EMAP) composta por, pelo menos, mais três profissionais (fisioterapeuta, assistente social, nutricionista, fonoaudiólogo, odontólogo, farmacêutico, terapeuta ocupacional ou psicólogo). ${ }^{11}$

É visto que a prática de $\mathrm{AD}$ reduz a demanda por atendimento em hospitais, promovendo a rotatividade e a disponibilidade dos leitos para casos de maior gravidade, e otimiza os custos com os serviços de saúde, sobretudo na saturada rede pública. ${ }^{5}$ Além do mais, a adesão ao tratamento é maior quando fora de um leito hospitalar, assim como possibilita a opção do óbito em casa, mas a decisão pela $\mathrm{AD}$ não depende apenas da vontade do enfermo e/ou família, mas também das condições clínicas e do contexto familiar. ${ }^{12}$ Pois, embora a intenção de humanização seja o foco, os seus cuidadores podem ficar sobre- 
carregados e se sentirem impotentes para lidar com as situações de dor e desconforto ao longo do processo até o estado de terminalidade do paciente, sendo fundamental que sejam orientados e acolhidos por uma equipe capacitada e habilitada para um enfrentamento com o mínimo de prejuízos físicos, psicossociais e espirituais. ${ }^{12}$

\section{CONSIDERAÇões FINAIS}

A complexidade dos pacientes paliativos somada a uma precária estrutura de saúde pública e também ao despreparo e/ ou desinteresse por parte dos profissionais e instituições de saúde refletem diretamente sobre a situação do CP no Brasil que ainda caminha com dificuldades, mas vão de encontro aos seus limites para trazer apoio e esperança de uma morte digna para os desamparados. A prática paliativa se faz presente do diagnóstico ao luto, podendo durar dias, meses ou até anos, e vem se expandindo para além do âmbito hospitalar, rumo aos atendimentos em ambulatórios e domicílios, mas ainda requer ampliação e descentralização de seus serviços. A AD também vem alcançando os lares de muitas famílias adeptas à causa, seja por meio do programa Melhor em Casa oferecido pelo SUS ou por instituições privadas, ao passo que a desospitalização prevê não somente a otimização dos custos em saúde, mas também uma alternativa de atenuar os efeitos deletérios de uma doença degenerativa.

Diferentemente de locais onde existem uma sólida estrutura para promoção de CP, tais como América do Norte, Europa e Japão, as pesquisas sobre a temática no contexto brasileiro ainda são escassas e de publicação recente, uma vez que a paliação ainda está restrita em locais onde se concentram os maiores interesses e investimentos financeiros. A modalidade de home care é dita como uma prática inovadora, promissora e ainda mais desafiadora para os profissionais habituados com o ambiente hospitalar. Mas de maneira geral, é visto a necessidade de rever os currículos de ensino em saúde, incentivar o aprimoramento dos profissionais em exercício, desenvolver e compartilhar estudos baseados em evidências que elevariam e levariam o CP e/ou a AD para o conhecimento e benefício de muitas vidas.

Não se trata de substituir os métodos convencionais, mas de complementar com recursos mais aceitáveis pelo paciente e, portanto, de maior adesão, mesmo quando a cura é inviável. A dor pode não diminuir, mas pode se tornar suportável a partir do momento em que o enfermo se encontra novamente em seu ambiente, amparado por seus entes queridos e profissionais aptos para promover os cuidados enquanto houver existência.

\section{REFERÊNCIAS}

1. Academia Nacional De Cuidados Paliativos. O que são Cuidados Paliativos [Internet]. 2009 [Acesso em: 2017 maio 07]. Disponível em: http://www.paliativo.org.br/ancp.php?p=oqueecuidados.

2. González JS, Ruiz MCS. A história cultural do cuidado paliativo em sociedades primitivas: uma revisão integrativa. Rev Esc Enferm USP [Periódicos na internet]. 2012 [Acesso em: 2017 abr 16]; 46 (4). Disponível em: http://www.scielo.br/pdf/reeusp/v46n4/33.pdf 
3. Gomes ALZ, Othero MB. Cuidados Paliativos. Estudos Avançados [Periódicos na internet]. 2016 [Acesso em: 2017 Maio 15]; 30 (88). Disponível em: http://www.scielo.br/scielo.php?script=sci arttext\&pid=S0103-40142016000300155\&lng=en\&nrm=iso

4. Neto G (Org.). Manual de Cuidados Paliativos. 2 ed. Lisboa: Faculdade de Medicina da Universidade de Lisboa; 2010.

5. Rodriguez MIF. Internação domiciliar: avaliações imperativas implicadas na função de cuidar. Psic. Rev.São Paulo [Periódicos na internet]. 2013 [Acesso em: 2017 Maio 14]; 2 (22). Disponível em: https://revistas.pucsp.br/index.php/psicorevista/article/view/17989

6. Oliveira AC, Silva MJP. "Autonomia em cuidados paliativos: conceitos e percepções de uma equipe de saúde". Acta paul. enferm. [Periódicos na internet]. 2010 [Acesso em: 2017 abr 24]; 23 (2). Disponível em: http://www.producao.usp.br/bitstream/handle/BDPI/4017/art OLIVEI$\underline{\text { RA Autonomia em cuidados paliativos conceitos e percepcoes 2010.pdf? sequence }=1}$

7. Cremesp. Cuidado Paliativo [Internet]. São Paulo: CREMESP, 2008 [Acesso em: 2018 Maio 30]. Disponível em: http://www.4estacoes.com/pdf/livros digitalizados/livro cuidado paliativo crm.pdf\#page $=15$

8. Brasil. Ministério da Saúde. Cadastro Nacional dos Estabelecimentos de Saúde [Homepage na internet]. 2018 [Acesso em: 2018 Maio 31]. Disponível em: http://cnes2.datasus.gov.br

9. Nickel Luana, Oliari LP, Vesco SNPD, Padilha MI. Grupo de pesquisa em cuidados paliativos: a realidade brasileira de 1994 a 2014. Esc Anna Nery [Periódicos na internet]. 2016 [Acesso em: 2018 Maio 28]; 20 (1). Disponível em: http://www.scielo.br/pdf/ean/v20n1/1414-8145ean-20-01-0070.pdf

10. Temel JS, Greer JA, Muzikansky A, Gallagher ER, Admane S, Jackson VA, et al. Early Palliative Care for patients with metastatic non-small-cell lung cancer. N Engl J Med [Periódicos na internet]. 2010 [Acesso em: 2017 Maio 10]; 363 (8). Disponível em: https://www.ncbi.nlm.nih.gov/ pubmed/20818875

11. Brasil. Ministério da Saúde - Portal da Saúde. Melhor em casa - Serviço de Atenção Domiciliar [Homepage na internet]. 2013. Acesso em: 24 Set 2017. Disponível em: http://portalsaude.saude. gov.br/index.php/cidadao/acoes-e-programas/melhor-em-casa

12. Sociedade Brasileira De Geriatria E Gerontologia. Vamos falar de cuidados paliativos? [Homepage na internet]. 2014 [Acesso em: 2018 Maio 30]. Disponível em: https://sbgg.org.br//wp-content/uploads/2014/11/vamos-falar-de-cuidados-paliativos-vers--o-online.pdf

\section{DADOS DOS AUTORES}

\section{Poliana Yabuuti}

Graduada em medicina pela Universidade Mogi das Cruzes (UMC), Mogi das Cruzes/SP - Brasil. Graduada em enfermagem pela Universidade Estadual de Londrina (UEL), Londrina/PR - Brasil. Residência em Gerência de Serviços de Enfermagem pela UEL Universidade Estadual de Londrina (UEL), Londrina/PR - Brasil. Pós graduanda em Medicina de Emergência pela UniAmerica Centro Universitário, Foz do Iguaçu/PR - Brasil. Atualmente é médica da equipe de Clínica Médica e Médica Hospitalista no Hospital Ipiranga de Mogi das Cruzes/SP - Brasil. poli_luri@yahoo.com.br

\section{Gustavo Monteiro de Jesus}

Graduação em medicina pela Universidade Mogi das Cruzes (UMC), Mogi das Cruzes/SP - Brasil. gustavo.jesus40@gmail.com 


\section{Maria Alice Santos Rocha}

Graduação em enfermagem pela Universidade Mogi das Cruzes (UMC), Mogi das Cruzes/SP - Brasil.m.alicesrocha@gmail.com

\section{KAREN EGÊA BACO}

Graduanda em fisioterapia pela Universidade Mogi das Cruzes (UMC), Mogi das Cruzes/SP - Brasil. karenegea@ymail.com

\section{Maria Olivia Costa Parra Rebolo}

Graduada em medicina pela Universidade Estadual de Londrina (UEL), Londrina/PR - Brasil. Médica da equipe de clínica médica do Hospital das Clínicas Luzia Pinho de Melo, Mogi das Cruzes/ SP - Brasil. Extensão universitária em Aperfeiçoamento em Cuidados Paliativos pelo Instituto Sírio Libanês de Ensino e Pesquisa, São Paulo/SP - Brasil. Residência em Clínica Médica pela Universidade Mogi das Cruzes (UMC), Mogi das Cruzes/SP - Brasil. marirebolo1@gmail.com

Submetido em: $18-8-2018$

Aceito em: 16-10-2021 